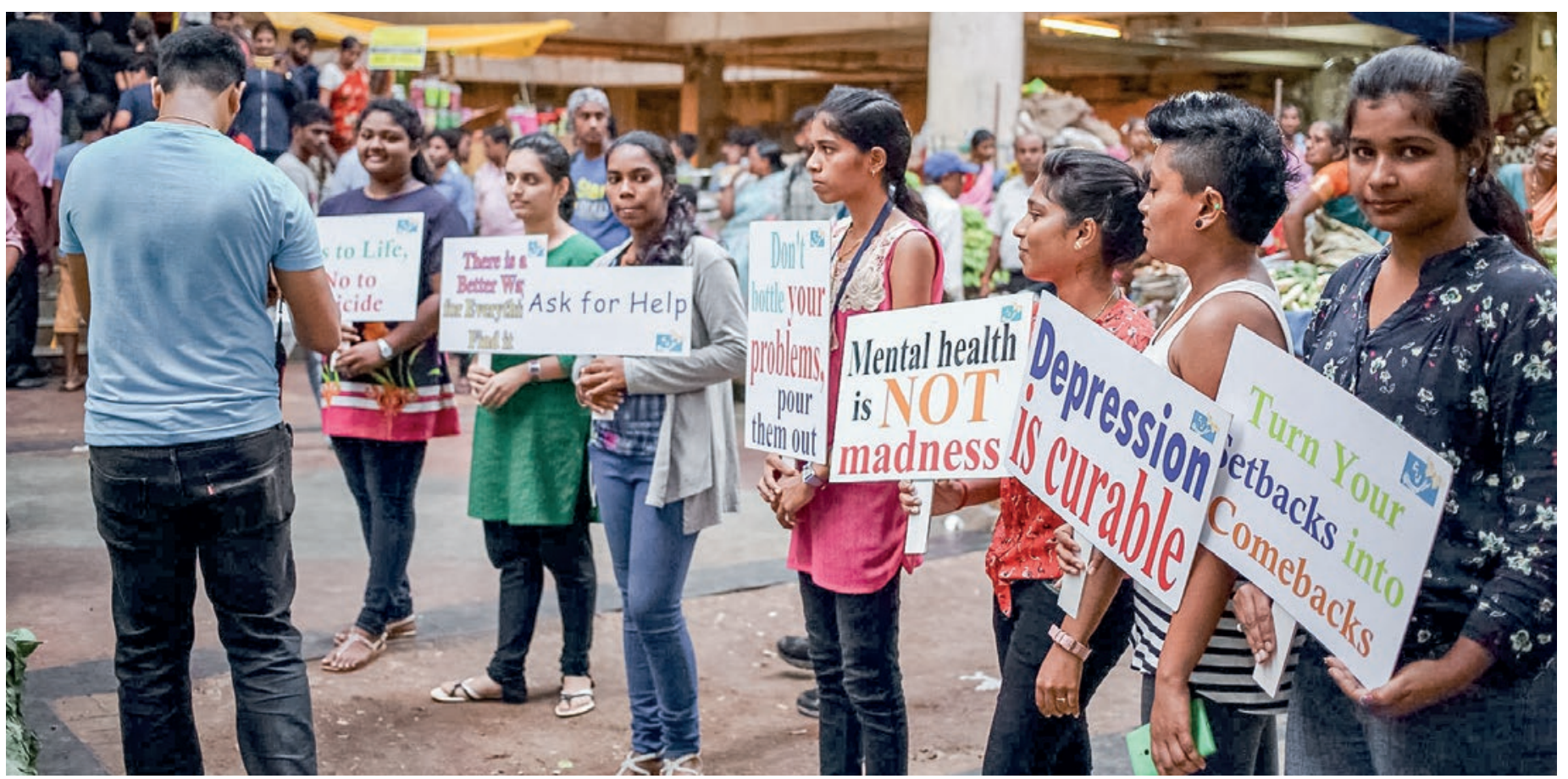

Delta facilite les soins professionnels à l'intention des personnes souffrant d'un trouble psychique et vivant dans des pays à ressources limitées comme l'Inde.

\title{
Une vision globale de la prise en charge des troubles psychiques
}

Catrina Mugglina, Gabriel Thorens ${ }^{b}$, Daniele Zullinoc, Peter Castelinod, Livia Winzeler ${ }^{\mathrm{e}}$, Thomas Müller ${ }^{\mathrm{f}}$, Conrad Frey $^{g}$, Justina Rackauskaite ${ }^{\text {h }}$, Barbara Hochstrasseri, Stefan KIöppeli, Sebastian Waltherk, Monika Müller pour delta - develop life through action

a Dr méd. Dr phil. nat., médecin cantonale du canton de Soleure*; b Dr méd., médecin adjoint, service d'addictologie, Département de psychiatrie HUG Genève; ${ }^{c}$ Prof. Dr méd., médecin-chef, service d'addictologie, Département de psychiatrie HUG Genève; ${ }^{d}$ Dr méd., fondateur et directeur de COOJ Mental Health Foundation, Goa, Inde; ${ }^{e}$ lic. phil., psychologue spécialiste en psychothérapie, Hôpital universitaire de gérontopsychiatrie, Berne*; ${ }^{\dagger}$ Prof. Dr méd. Psychiatrie universitaire, Berne, directeur de la Clinique de psychiatrie privée de Meiringen**; ${ }^{9}$ Dr méd., médecin-chef émérite Psychiatrie Obwalden/ Nidwalden**; ${ }^{h}$ Cheffe de clinique, Psychiatrie Luzern; ' Dr méd., médecin-cheffe Clinique de psychiatrie privée de Meiringen**; j Prof. Dr méd., Hôpital universitaire de gérontopsychiatrie, Berne; ${ }^{k}$ Prof. Dr méd., Hôpital universitaire de psychiatrie et psychothérapie, Berne; ' Dr méd. Dr phil. nat., cheffe de clinique Hôpital universitaire de psychiatrie et psychothérapie, Berne, Visiting Lecturer Center for Global Mental Health, King's College London*

Comité directeur de Delta ** Conseil consultatif de Delta

La santé mentale est hautement importante et ne doit pas être négligée. Cet article se concentre sur le contexte global des troubles psychiques et constate que les soins sont insuffisants dans les pays à ressources limitées. L’initiative suisse «Delta - develop life through action" a pour mission de faciliter l'accès pour les personnes concernées à des soins professionnels et spécialisés dans ces pays.

Importance globale des troubles psychiques

L'évaluation du Global Burden of Disease Study montre clairement que les troubles psychiques progressent dans les pays à ressources limitées et que le handicap associé est préoccupant [1]. Mondialement, 14\% des Years Lived with Disability sont attribués aux troubles psychiques (hommes et femmes de tous les âges). Chez les personnes en âge de travailler, la dépression est la deuxième cause de diminution de la qualité de vie et le suicide la troisième cause de mortalité. Dans l'absolu, la plupart des personnes concernées vivent dans des pays du Sud à ressources limitées. La recherche a par ailleurs démontré que la prévalence des troubles psy- 
chiques dans ces pays était comparable à celle des pays industrialisés et il faut souligner que la majorité de la population mondiale vit dans ces régions [2]. En plus de souffrir de problèmes de santé, les personnes concernées sont fréquemment victimes de violation des droits humains. Leurs droits civils sont niés par des mesures tutélaires et souvent, elles sont placées dans des institutions (y compris des prisons) contre leur volonté, sans mandat de traitement clair. En plus, les personnes atteintes de maladies psychiques vivent dans une grande précarité sociale et se retrouvent souvent sans-abris [3]. Les conséquences des troubles psychiques ne concernent pas seulement les personnes touchées, mais la société entière. Une publication ré-

Dans l'absolu, la plupart des personnes atteintes de maladies psychiques vivent dans des pays du Sud à ressources limitées.

cente a évalué le bénéfice économique en cas de traitement adéquat de la dépression et des troubles anxieux [4]. Le bénéfice, représenté par l'aptitude de travail récupérée et une augmentation de la productivité grâce à une thérapie adéquate, dépasse plusieurs fois les coûts de traitement. Les auteurs montrent que cette association existe autant pour les pays avec des ressources limitées que pour les pays industrialisés. Néanmoins, les personnes souffrant de troubles psychiques ne sont que très peu prises en charge dans les pays à ressources limitées.

\section{Traitement des troubles psychiques: pays à ressources limitées mal lotis}

Selon l'OMS, les pays avec des ressources limitées ne prennent pas en compte l'augmentation de la demande pour des structures de soins professionnels. Le Mental Health Atlas détaille les ressources mondiales dans le domaine des soins psychiatriques [5]. Entre 50\% et $90 \%$ des personnes concernées ne reçoivent pas de traitement adéquat dans les pays à ressources limitées. Par exemple, dans les pays industrialisés, un patient sur cinq souffrant d'un épisode dépressif sévère reçoit un traitement adéquat minimal. Dans les pays à ressources limitées, seul un patient sur 27 est traité [6]. Dans ces pays, un psychiatre est responsable d'un bassin de deux millions de personnes (dans les pays industrialisés, c'est un psychiatre par 11500 habitants [5, 7]). Ces pays dépensent en moyenne 0,25 dollar par habitant et par année pour le traitement des troubles psychiques, correspondant à $1 \%$ des toutes les dépenses de santé $[5,7]$. Les troubles psychiques sont donc les problèmes de santé les plus négligés dans les pays avec des ressources limités. De plus, la communauté internationale échoue largement à garantir un financement adéquat des programmes de prévention, de thérapie et de réhabilitation des troubles psychiques. Les fonds de développement ne dépensent que 0,85 dollar par DALY (Disability-adjusted life year) pour les troubles psychiques - en comparaison avec les 144 dollars par DALY pour le VIH/SIDA et les 48 dollars par DALY pour la tuberculose et la malaria [8, 9]. La conséquence de l'incapacité des gouvernements à garantir des soins adéquats est que les personnes concernées couvrent elles-mêmes la plupart des frais de traitement et d'encadrement. Cela accentue la précarité des soins car les personnes souffrant d'un trouble psychique vivent souvent aussi dans la pauvreté et n'ont pas les moyens financiers pour couvrir les dépenses nécessaires à un traitement approprié. Dans ce contexte, l'importance des organisations non gouvernementales (ONG) dans le futur de la santé mentale devient évidente.

\section{Volonté de faciliter l'accès aux soins dans les pays à ressources limitées}

Delta - develop life through action, comme association bénévole suisse, facilite les soins professionnels et spécialisés à l'intention des personnes souffrant d'un trouble psychique et vivant dans des pays à ressources limitées. Nous nous engageons dans la formation des professionnels de santé locaux; nous couvrons les dépenses des traitements pour les patients qui n'ont pas les moyens financiers pour les couvrir eux-mêmes; nous soutenons des institutions dans le développement des services de soins et nous encourageons l'intégration des personnes concernées dans la société et dans la reprise d'un travail.

Actuellement, nous réalisons quatre projets dans le sud de l'Inde en collaboration avec nos partenaires locaux. A titre d'exemple, nous avons reçu le Prix Perspectives pour la collaboration avec la fondation $\mathrm{COOJ}$ Mental Health Foundation à Goa. Ce prix de parrainage encourage depuis quinze ans des projets innovants centrés sur les personnes concernées et leur entourage pour leur ouvrir de nouvelles perspectives. Delta soutien la fondation COOJ depuis 2016 pour établir un service de psychiatrie gériatrique, initialement en développant une clinique de jour et maintenant le Geriatric Digital Intervention Program. Le deuxième en réponse à la pandémie de Covid-19 fortement présente en Inde. L'écart de traitement préexistant s'est amplifié en raison de la pandémie, et cela surtout pour les personnes âgées avec des troubles psychiques et cognitifs. Les personnes âgées, comme population à risque, sont souvent confinées au domicile par les mesures sani- 
taires contre le Covid-19, avec une augmentation de l'isolement social comme conséquence. Cette dernière a un impact majeur sur la santé en général et sur la santé mentale en particulier et est considérée comme facteur de risque comparable au tabagisme. De plus, les options thérapeutiques de proximité et les offres digitales manquent grandement en Inde. Ce sont exactement ces éléments thérapeutiques qui sont indispensables pour le maintien des soins professionnels pendant des périodes de confinement prolongé. En même temps, on a constaté qu'un cadre thérapeutique exclusivement digital s'est avéré défavorable. Les outils digitaux ne sont pas familiers aux personnes âgées, avec comme résultat un manque de compétences techniques nécessaires. Avec notre Geriatric Digital Inter-

\section{Delta facilite les soins à l'intention des per- sonnes souffrant d'un trouble psychique et vivant dans des pays à ressources limitées.}

vention Program, nous prenons en compte cette problématique. Les personnes âgées reçoivent une combinaison de thérapie digitale et de proximité pour rendre possibles des soins spécialisés dans leur environnement familial. Le programme thérapeutique s'oriente vers le principe de task-shifting. En raison du manque de thérapeutes spécialisés, certains éléments thérapeutiques sont délégués à des personnes non professionnelles [10]. La recherche a clairement démontré que ces personnes sont aptes à traiter les troubles psychiques les plus fréquents si elles sont formées et supervisées correctement. Cela favorise une utilisation efficace des ressources limitées en augmentant l'accès aux traitements pour les troubles psychiques les plus courants et en permettant au personnel spécialisé de

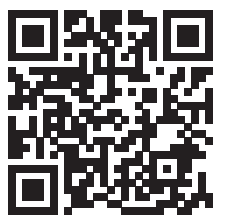

Lien vers le webinar "Global Mental Health"

Monika Müller Association Delta-develop life through action CH-3000 Berne monika.mueller[at] delta-ngo.ch

\section{L'essentiel en bref}

- Les maladies mentales sont en augmentation dans le monde entier et les disparités sanitaires qui en résultent sont importantes. Dans le même temps, jusqu'à $90 \%$ des personnes touchées dans les pays pauvres en ressources ne reçoivent pas de traitement adéquat.

- Delta - develop life through action est une association suisse qui assure le traitement professionnel des malades mentaux dans ces pays.

- En particulier dans le contexte de la pandémie de Covid-19, Delta collabore avec la Fondation COOJ pour la santé mentale à Goa, en Inde. Le Geriatric Digital Intervention Program a été développé. se concentrer sur les patients avec des troubles plus sévères. Les digital buddies aident les patients à se repérer dans le monde digital. Ce sont des personnes non professionnelles qui ont neuf ans d'études et qui reçoivent pendant un mois une formation intense à la fondation COOJ pour se préparer à leurs tâches. Premièrement, notre but est d'améliorer les compétences digitales des personnes âgées souffrant de troubles psychiques ou cognitifs afin de faciliter un cadre thérapeutique avec des éléments digitaux. Deuxièmement, elles peuvent entraîner leurs stratégies cognitives (remédiation cognitive, entraînement de la mémoire, capacité de résolution de problème) grâce à différentes applications installées sur des tablettes, avec comme but ultérieur d'améliorer leur vie quotidienne. Le Prix Perspectives nous permet d'évaluer l'acceptabilité et la faisabilité de ce projet dans le contexte indien.

Pour plus d'informations sur l'association Delta et son travail, ainsi que sur les possibilités de soutien, veuillez consulter le site: www.delta-ngo.ch.

\section{Crédits photo}

Programme de sensibilisation suicide de delta/COOJ à Goa, Inde

\section{Références}

1 Global Burden of Disease Collaborators. Global, regional, and national incidence, prevalence, and years lived with disability for 354 diseases and injuries for 195 countries and territories 1990-2017: a systematic analysis for the Global Burden of Disease Study. 2017. Lancet. 2018;392(10159)1789-858.

2 Steel Z, et al. The global prevalence of common mental disorders a systematic review and meta-analysis 1980-2013. International journal of epidemiology. 2014;43(2):476-93.

3 Beijer U, Andréasson S. Gender, hospitalization and mental disorders among homeless people compared with the general population in Stockholm. European Journal of Public Health 2010;20(5):511-6

4 Chisholm D, et al. Scaling-up treatment of depression and anxiety: a global return on investment analysis. Lancet Psychiatry. 2016;3(5):415-24.

5 WHO, Mental Health Atlas 2017. World Health Organisation. Geneva 2017.

6 Thornicroft G, et al. Undertreatment of people with major depressive disorder in 21 countries. Br J Psychiatry. 2017;210(2):119-24.

7 Saxena S, et al. Resources for mental health: scarcity, inequity, and inefficiency. Lancet. 2007;370(9590):878-89.

8 Patel V, et al. The Lancet Commission on global mental health and sustainable development. Lancet. 2018;392(10157):1553-98.

9 Charlson FJ, et al. Donor Financing of Global Mental Health, 19952015: An Assessment of Trends, Channels, and Alignment with the Disease Burden. PLoS One. 2017;12(1):e0169384.

10 Purgato $\mathrm{M}$, et al. Promotion, prevention and treatment interven tions for mental health in low- and middle-income countries through a task-shifting approach. Epidemiol Psychiatr Sci. 2020;29:e150. 